\title{
Spatial frequency content of visual imagery
}

\author{
SETH KUNEN and JAMES G. MAY \\ University of New Orleans, Lakefront, New Orleans, Louisiana 70122
}

\begin{abstract}
Three experiments employing the McCollough paradigm were conducted to determine the spatial-frequency content of visual imagery. In Experiment 1, large and reliable patterncontingent color aftereffects were obtained after adaptation to visual imagery. The direction of the aftereffects indicated that subjects were adapting to higher spatial frequencies in their imagery. These results contrast with the data of Experiment 2, which demonstrate that color aftereffects obtained with adaptation to physically present stimuli are mediated by the fundamental spatial frequency components. The magnitude of the imagery-induced aftereffects in Experiment 1 equaled the magnitude of the externally induced aftereffects obtained in Experiment 2 with the same subjects. By blurring the to-be-imaged patterns (Experiment 3), the fundamental Fourier components became the salient perceptual features of the stimuli, and the direction of the imagery-induced aftereffects was reversed from that of Experiment 1, indicating that the spatial frequency content of the imagery had changed from higher to lower frequencies. Under normal viewing conditions, subjects use the higher spatial frequencies associated with the perceptually salient edges of stimuli to construct their images. The results of Experiments 1 and 3 are discussed in light of a current controversy over the nature of information representation in imagery, and it is concluded that support has been obtained for the analog model of visual imagery.
\end{abstract}

McCollough (1965) was the first to demonstrate orientation-contingent color aftereffects. In her study, subjects first viewed alternating horizontal and vertical lines on different chromatic backgrounds, and then were shown adjacent panels containing the same horizontal and vertical lines on a white background. The subjects reported seeing on each test pattern the color complementary to the color originally associated with that pattern. Finke and Schmidt $(1977,1978)$ recently reported the fascinating finding that the visual aftereffects obtained in the McCollough paradigm could also be obtained by instructing subjects to visually imagine bar gratings on either patterned or unpatterned homogeneous chromatic backgrounds.

The Finke and Schmidt experiments raised two intriguing questions that we attempted to answer. First, since they used verbal report measures, they could not measure the magnitude of the perceptual aftereffects. Could the perceptual aftereffects be more objectively measured using different psychophysical techniques? Second, since the high and low spatial frequency components of the stimuli employed by Finke and Schmidt had identical orientations, they could not determine which spatial frequency components mediated such visual imagery effects. Were the perceptual aftereffects obtained with visual imagery derived from the same spatial frequency components of complex stimuli as were aftereffects obtained with externally present visual stimuli?

Previous studies (Green, Corwin, \& Zemon, 1976; May, Agamy, \& Matteson, 1978; May \& Matteson, 1976; May, Matteson, Agamy, \& Castellanos, 1978) had established that, when checkerboards with spatial frequencies above 3.0 cycles/deg were used as stimuli, McCollough effects were derived from adaptation to the fundamental spatial frequency components. If the same spatial frequency components in actual stimuli were contained in imagined stimuli, then adaptational effects might be mediated by the fundamentals in imagined checkerboards.

\section{EXPERIMENT 1}

\section{Method}

Subjects. Eight subjects participated in the study. They ranged in age from 18 to 37. All had normal color vision and 20/20 visual acuity. With one exception, none of the subjects had previously experienced the McCollough effect, nor were they aware of the hypothesis under investigation.

Stimuli and Apparatus. The adaptation stimuli consisted of oblique checks (called diamonds) and nonoblique checks (called squares). These achromatic stimuli were projected on a plain white background. The checkerboard stimuli had a fundamental spatial frequency of $3.0 \mathrm{cycles} / \mathrm{deg}$, and thus the diagonal of each check subtended $20 \mathrm{~min}$ of visual angle. The contrast of the stimuli was $95 \%$, and they were presented by Kodak Carousel slide projectors in a 12-deg square field at a space-average luminance of $9.4 \mathrm{~cd} / \mathrm{m}^{2}$. The checkerboards were selected for use as adaptation stimuli because Kelly (1976) had shown that the twodimensional Fourier analysis of such patterns contained fundamental spatial frequency components oriented at 45-deg angles to the edges of individual checks and that the odd harmonic spatial components were distributed throughout meridians that were different from those of the fundamentals or the edges. Although none of the spatial frequency components were aligned in exactly the same orientation as the edges, the higher harmonics did contribute to clarity of edge perception. They were also chosen because recent studies had shown that, with stimuli having the same spatial frequency as our stimuli $(3.0$ cycles $/ \mathrm{deg})$, orientationcontingent color aftereffects were reliably associated with the fundamental Fourier components of such stimuli. The alternating 
magenta and green fields used during adaptation were produced by mounting Kodak filters (Wratten No. 32 and No. 53, respectively) in two Kodak projectors equipped with individual shutters.

The test apparatus has been fully described by May et al. (1978). In outline, the subject inspected two halves of a visual test field ( $8 \mathrm{deg}$ horizontal) that contained vertical and oblique rectilinear gratings of identical spatial frequency. A colorcompensating filter was rotated to obtain a chromatic match between the two halves of the test field. The test stimuli had a contrast of $65 \%$ and were presented at a luminance level of $4.3 \mathrm{~cd} / \mathrm{m}^{2}$. The fundamental spatial frequency of the test stimuli was the same as that of the adaptation stimuli $(3.0 \mathrm{cycles} / \mathrm{deg})$. Thus, the bar widths subtended 10 min of visual angle.

Procedure. Each of the eight subjects was tested individually, using previously described methods (for full details, see May et al., 1978). The testing session began with the subjects adjusting the filter to obtain a chromatic match between the two halves of the test field. Specifically, we instructed the subjects to "rotate the knob until both halves of the stimulus field are the same color." Adjustment of the color-cancellation filter varied the degree of colorimetric purity in the adjacent test field by allowing magenta light to be added to one grating while green light was added to the other grating. Subjects fixated a point in the exact middle of the pattern while making the matches. Ten matches were made, five beginning on each side of the match point. The distance from the match point for each match and the starting direction of the set of matches were randomized for each subject. The data were collected by an experimenter who had no knowledge of the spatial frequency relationships in the stimuli used. This preadaptation data yielded individual baselines against which the color adaptation aftereffects could be compared. The use of the color cancellation procedure minimized the influence of response biases often associated with verbal report measures.

After obtaining the baseline data, the subjects were told that the next phase would involve visually imaging two checkerboard patterns and that they would have $2 \mathrm{~min}$ to study the patterns. The subjects were then given 2 min of preadaptation familiarization with the achromatic patterns, which were alternately exposed to the subjects at the rate of $5 \mathrm{sec}$ per pattern, with an interstimulus interval of less than $250 \mathrm{msec}$. Immediately after the 2 min of familiarization, the subjects were told that the patterns would be replaced with alternating homogeneous magenta and green fields and that they would be expected to visualize the diamonds (or squares) on the magenta and the squares (or diamonds) on the green. The subjects were instructed to replace the actual checkerboards with their visually imagined checkerboards with as much fidelity as possible. Half the subjects were instructed to visualize the squares on magenta and the diamonds on green, and the other half were instructed to visualize the diamonds on magenta and the squares on green. The magenta and green fields alternated at a $5-\mathrm{sec}$ rate. Midway through the imagery session, the achromatic diamonds and squares were alternately reexposed three times at a 5 -sec rate to refresh the subjects' memories. At no time during adaptation were the checkerboard stimuli actually presented in chromatic light. After the $15 \mathrm{~min}$ of "internal" adaptation, the subjects performed another 10 colorimetric matches as in the preadaptation procedure.

By rotating the set of color-compensating filters, the observer could offset his colored aftereffects by adding complementary colors to either side of the test field. If the subject was adapting to fundamentals in his imagery in the magenta-diamonds/greensquares condition, he would add green to the oblique stripes and magenta to the vertical stripes to achieve a match. This coloraddition process would be reversed in the green-diamonds/magentasquares condition. If, on the other hand, the subject was adapting to higher spatial frequencies in his imagery, he would add magenta to the vertical stripes and green to the oblique stripes in the greendiamonds/magenta-squares condition, and the reverse in the magenta-diamonds/green-squares condition. When the subjects' adjustments indicated adaptation to fundamentals, we designated such scores "positive" to distinguish them from effects derived from adaptation to higher spatial frequencies, which we designated "negative."

\section{Results and discussion}

The dependent variable was the mean difference score, which was computed by subtracting the mean of preadaptation match points from the mean of postadaptation match points for each subject (see Figure 1). The mean imagery scores were significantly more negative than zero $[\mathrm{t}(7)=4.73, \mathrm{p}<.01 ; \alpha=.05]$. The significant imagery-induced aftereffects verify the reports of Finke and Schmidt that McCollough aftereffects can be derived from adaptation to visual imagery and, furthermore, that these aftereffects can be objectively measured using psychophysical techniques. The imagery aftereffects are quite striking, since they provide the first data, that we are aware of, demonstrating the spatial frequency content of visual imagery. Surprisingly, and contrary to our ex-

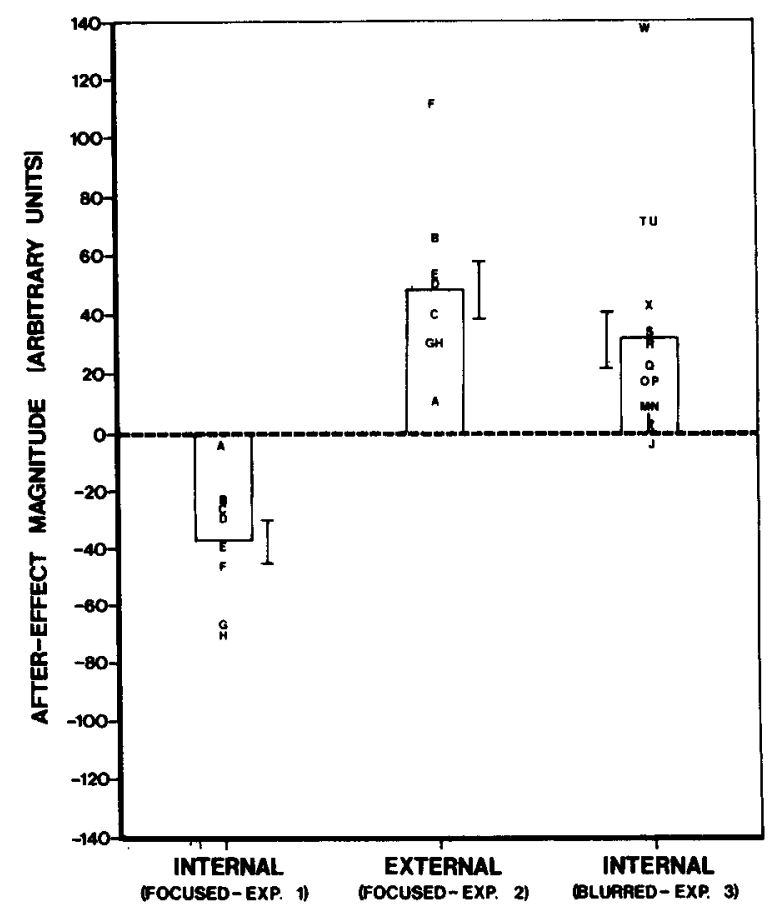

ADAPTATION CONDITION

Figure 1. Magnitude of colored aftereffects following adaptation to internally generated visual stimulation after viewing sharply focused checkerboards (Experiment 1), externally presented sharply focused checkerboards (Experiment 2), and internally generated visual stimulation after viewing blurred checkerboards (Experiment 3). The arbitrary units on the ordinate represent the angular deviation between the sets of cross-Polaroids in the test apparatus and are proportional to excitation purity. Negative scores represent aftereffects derived from adaptation to higher spatial frequencies associated with edges, while positive scores indicate adaptation to fundamental Fourier components. Means and standard errors are indicated. 
pectations, the data indicate that our subjects were adapting, not to fundamentals as subjects do when they adapt to externally present checkerboards, but to the higher spatial frequencies.

In Experiment 2, we compared the magnitude of the color aftereffects obtained from adaptation to actual squares and diamonds superimposed on either the magenta or green backgrounds with the same subjects. At least 1 week intervened between the two experiments. Since we did not know how long imageryinduced aftereffects would last, we repeated 10-trial tests on a daily basis with selected subjects. The adaptation effects did last as long as a week for some subjects and as long as 3 weeks for one subject. When each subject's postadaptation scores had returned to the baseline, he was permitted to participate in the external adaptation experiment.

\section{EXPERIMENT 2}

\section{Method}

Subjects and Procedure. The subjects of Experiment 1 were asked to participate in a second similar study. All eight subjects agreed to return for Experiment 2. The basic procedure of Experiment 1 was followed in Experiment 2. The major difference between the first and second experiments was that the familiarization phase of the first experiment was omitted and subjects adapted for 15 uninterrupted minutes to the alternating squares and diamonds on the chromatic backgrounds. To prevent the formation of afterimages, the subjects were instructed during adaptation to allow their gaze to shift about the middle two-thirds of the checkerboard patterns. As in the first experiment, subjects were asked to make 10 pre- and 10 postadaptation colorimetric matches with the same stimuli. The pattern-color combinations were determined randomly for each subject in the adaptation phase.

\section{Results and Discussion}

The dependent variable was the same mean difference score used in Experiment 1 (see Figure 1). The mean difference scores were significantly more positive than zero $[\mathrm{t}(7)=4.50, \mathrm{p}<.01 ; \alpha=.05]$. The absolute value of the magnitude of the color aftereffects induced by external stimulation was not reliably different from that induced by visual imagery [correlated $\mathrm{t}(7)=.87, \mathrm{p}<.20$. $^{2}$

The adaptation pattern that resulted from the physically present stimuli is the same as that obtained in previous McCollough experiments using the same stimuli (May \& Matteson, 1976; May et al., 1978), in which adaptation has been shown to be derived from the fundamental Fourier components. The imagery-induced aftereffects are not derived from the fundamental Fourier components, but from adaptation to the higher harmonics associated with the edges of the patterns. When a sharply focused checkerboard of medium spatial frequency ( 3.0 cycles/ deg) is examined, it is the edges of the checks that are the phenomenally prepotent aspects of the pattern. Subjects are not aware of the fundamental Fourier components unless the higher harmonic com- ponents are filtered out, as occurs with defocused patterns. The imagery generated by our subjects appears not to contain the fundamental Fourier components present in the external stimuli, since the aftereffects obtained in the imagery condition are the same as would occur if only the higher harmonics were present.

Apparently, subjects construct their visual images from the higher spatial frequencies associated with the visually salient edges of the stimuli. This finding naturally raises the question as to whether subjects are capable of representing fundamental spatial frequency components in their visual imagery. To test whether subjects can, in fact, represent fundamentals in their imagery, we designed a third experiment. We reasoned that if the higher spatial frequencies associated with edges of the checks were responsible for the aftereffects obtained with internal adaptation, then it should be possible to reverse the adaptation pattern obtained with internal adaptation by decreasing the salience of the higher spatial frequencies while increasing the salience of the fundamental Fourier components. This can be accomplished by blurring the pattern to be imaged so that higher spatial frequencies are filtered out while the fundamentals become more perceptually salient. After viewing blurred patterns, subjects should construct images that contain primarily fundamentals, which should, in turn, produce adaptation aftereffects opposite to those obtained in Experiment 1.

\section{EXPERIMENT 3}

\section{Method}

Subjects. The third experiment was conducted with 14 new subjects recruited from undergraduate psychology courses. None of the subjects had previously experienced the McCollough effect, and none were aware of the hypothesis being tested. All subjects had $20 / 20$ vision, or vision corrected to $20 / 20$ with noncolored contact lenses.

Procedure. The procedure of the third experiment was the same as that of the first experiment, with two exceptions. In the familiarization phase, the subjects viewed the checkerboard patterns through +1.75-diopter spherical lenses. These lenses defocus the stimuli so that the higher spatial frequencies associated with the edges are attenuated while the fundamentals are accentuated. At no time did the subjects view the patterns without the lenses. Also, the subjects were asked to verbally describe what they were viewing during the familiarization phase so that we could be sure that they were, in fact, noticing the fundamentals in the checkerboards

\section{Results and Discussion}

The difference scores resulting from this experiment are depicted as the third column in Figure 1. The mean difference scores were significantly more positive than zero $[\mathrm{t}(13)=3.07, \mathrm{p}<.01 ; \alpha=.05]$, but were not significantly different in magnitude from the aftereffects of Experiment $1[\mathrm{t}(20)=.52]$ or Experiment 2 $[\mathrm{t}(20)=1.23]$.

Subjects in Experiment 3 were asked to image pat- 
terns in which the fundamentals and not the higher harmonics were the salient perceptual features. When the subjects imaged such patterns, the direction of their aftereffects reversed compared with those obtained in Experiment 1. The results of Experiment 3 replicate the findings of Experiment 1 that visual imagery can be used to induce color aftereffects, and in addition, by reversing the direction of the aftereffects, provide evidence that subjects in Experiment 1 were, in fact, adapting to the higher spatial frequencies in their imagery.

\section{GENERAL DISCUSSION}

Apparently, subjects do not attend to the fundamentals of 3.0-cycles/deg checkerboard stimuli under normal viewing conditions and, consequently, do not reproduce the fundamentals in their imagery. In Experiment 3, it was demonstrated that the absence of fundamentals in the imagery of the subjects of Experiment 1 was not due to a limitation in representation ability of the subjects. When the fundamentals were perceptually salient, the subjects were able to reproduce them instead of the higher spatial frequencies in their imagery.

The visual imagery of our subjects was highly veridical in other respects. Pattern-contingent aftereffects obtained with external adaptation have been shown to be quite specific to spatial frequency and orientation (Breitmeyer \& Cooper, 1972; Fidell, 1970; Stromeyer, 1972). The reliable aftereffects obtained with internal adaptation indicates that our subjects were able to quite accurately visualize patterns of the same orientation and size as they were instructed to image. Our subjects were also able to exercise a high degree of control over their images, since they were able to switch from one image to another within the time limits of our 5-sec alternation cycle.

These results also have relevance to a current controversy concerning the nature of information representation in visual imagery. One prominent position (analog model) holds that visual images contain information in a spatial format that is similar to the format of information contained in visual perception (Kosslyn \& Pomerantz, 1977). The propositional model, in contrast with the analog model, argues that all visual information is represented in an abstract, logical, nonmodality-specific code (Pylyshyn, 1973). Although some researchers (e.g., Anderson, 1978) believe that the two models may in practice be impossible to distinguish, others believe that the analog model can be distinguished from the propositional model by empirically fulfilling predictions that analog representations have properties intrinsic to the visual system (see Pylyshyn, 1979, p. 20). Evidence of such intrinsic properties has recently been reported by Finke and Kosslyn (1980), who demonstrated that fields of resolution in imagery and visual perception are nearly identical in shape, which suggests that there are some neural mechanisms common to both imagery and vision.

The present study also provides evidence that imagery and vision share certain neural processes. The extremely close correspondence between Experiments 2 and 3 suggests that the mechanisms responsible for internally and externally derived pattern-contingent color aftereffects are the same. Pattern-contingent aftereffects obtained from internal adaptation in Experiments 1 and 3 are entirely consistent with analogical predictions that imagery will, at some level, reveal properties that are typically unique to the visual system. On the other hand, the pattern-contingent aftereffects obtained in Experiments 1 and 3 are highly modality-specific effects that are inconsistent with the propositional model. It is difficult to understand which assumptions underlying the propositional model would lead to the prediction that internal adaptation would result in pattern-contingent colored aftereffects. The pattern-contingent aftereffects obtained in Experiments 1 and 3 provide evidence that imagery possesses some of the "intrinsic properties" of the visual system, which, according to Pylyshyn's argument, constitutes strong support for the analog model of imagery.

The present set of experiments uncovered significant new information concerning the nature of information representation in visual imagery. The performance of subjects in Experiments 1 and 3 supports the impression we received from introspective reports from subjects that unless a particular spatial frequency pattern is consciously detected, it could not be reproduced in their imagery. In contrast, with externally derived McCollough effects, subjects are not typically aware of the fundamental Fourier components to which they adapt in checkerboards. Although it appears that subjects need to be consciously aware of specific spatial frequency information before they can incorporate such information into their visual images, once the images are formed, subjects can adapt to their images much in the same fashion as they adapt to externally present stimuli. Visual images can apparently serve as functional continuations of visual perception and are shown to be potent sources of visual information that can systematically alter an individual's perception of the world.

\section{REFERENCES}

Anderson, J. R. Arguments concerning representations for mental imagery. Psychological Review, 1978, 85, 249-277.

Bre itmeyer, B. G., \& Cooper, L. A. Frequency-specific color adaptation in the human visual system. Perception \& Psychophysics, 1972, 11, 95-96.

Fidelt, L. S. Orientation specificity in chromatic adaptatinn of human "edge-detectors." Perception \& Psuchophysics, 1970. 8, 235-237. 
Finke, R. A., \& Kossiyn, S. M. Mental imagery acuity in the peripheral visual field. Journal of Experimental Psychology: Human Perception and Performance, 1980, 6, 126-139.

FinKe, R. A., \& SCHMidT, M. H. Orientation-specific color aftereffects following imagination. Journal of Experimental Psychology: Human Perception and Performance, 1977, 3, 599-606.

Finke, R. A., \& Schmidt, M. H. The quantitative measure of pattern representation in images using orientation-specific color aftereffects. Perception \& Psychophysics, 1978, 23, 515-520.

Green, M., Corwin, R. T., \& Zemon, Y. A comparison of Fourier analysis and feature analysis in pattern-specific color aftereffects. Science, 1976, 192, 147-148.

Jones, P. D., \& Holding, D. H. Extremely long-term persistence of the McCollough effect. Journal of Experimental Psychology: Human Perception and Performance, 1975, 1, 323-327.

KELLY, D. H. Pattern detection and the two-dimensional Fourier transformation: Flickering checkerboards and chromatic mechanisms. Vision Research, 1976, 16, 277-289.

Kosslyn, S. M., \& Pomerantz, J. R. Imagery, propositions, and the form of internal representations. Cognitive Psychology, $1977,9,52-76$.

May, J. G., Agamy, G., \& Matteson, H. H. The range of spatial frequency-contingent color aftereffects. Vision Research, $1978,18,917-921$.

MaY, J. G., \& Matteson, H. H. Spatial frequency-contingent color aftereffects. Science, 1976, 194, 145-147.

May, J. G., Matteson, H. H., Agamy, G., \& Castellanos, P. The effects of differential adaptation on spatial frequencycontingent color aftereffects. Perception \& Psvchophysics, 1978 , 23, 409-412.

McCollough, C. Color adaptation of edge-detectors in the human visual system. Science, 1965, 149(Whole No. 3688), 1115-1116.

Pylyshyn, Z. W. What the mind's eye tells the mind's brain: A critique of mental imagery. Psychological Bulletin, 1973, 80, $1-24$.

Pylyshyn, Z. W. The rate of "mental rotation" of images: A test of a holistic analogue hypothesis. Memory \& Cognition, $1979,7,19-28$.

Skowbo, D., Gentry, T., Timney, B., \& Morant, R. B. The McCollough effect: Influence of several kinds of visual stimulations on decay rate. Perception \& Psychophysics, 1974, 16, 47-49.

Stromeyer, C. F. Edge-contingent color aftereffects: Spatial frequency specificity. Vision Research, 1972, 12, 717-734.

White, K. D. Luminance as a parameter in establishment and testing of the McCollough effect. Vision Research, 1976, 16, 297-302.

\section{NOTES}

1. Since Finke and Schmidt were not concerned with differential adaptation to fundamentals or odd harmonics, they used the terms "positive" and "negative" to refer to perceptual afterimages in the traditional sense. Our present use of positive and negative scores should not be confused with positive and negative afterimages. When the respective axes of the cross Polaroids con- tained in the cancellation filter are rotated $45 \mathrm{deg}$ from the axes of their companion cross Polaroids associated with the stimulus patterns, the projected image of the test patterns contained equal amounts of magenta and green light. Whenever the axes of the cancellation and stimulus cross Polaroids were aligned, then one stimulus panel contained a maximum of green and a minimum of magenta, and vice versa, for the adjacent stimulus panel. We recorded the angular deviation between the axes of these sets of Polaroids to the nearest degree. Thus, the designation of positive and negative is arbitrary in the absolute sense and designates the direction of the aftereffects relative to the stimulus conditions during adaptation. The relationship between our scores (in units of angular deviation) and the excitation purity (Pe) of colors necessary to cancel out the aftereffects was approximately linear (see White, 1976). All matches were made within the limits of a restricted range of angular deviations, and the nonlinear effects were estimated as follows:

McClaurin's power series expansion of $\cos ^{2} \theta$ about $\theta=45 \mathrm{deg}$ $=\pi / 4$ radians; let $\theta=\pi / 4+\delta$, where $\delta$ (expressed in radians) is the largest angular deviation of $\theta$ from $\pi / 4$ radians.

$$
\begin{aligned}
\cos ^{2} \theta=\cos ^{2}(\pi / 4 & +d) \\
\cos ^{2}(\pi / 4+\delta)= & \cos ^{2} \pi / 4-(2 \cos \pi / 4)(\sin \pi / 4) \delta \\
& +2\left(\sin ^{2} \pi / 4-\cos ^{2} \pi / 4\right) \delta^{2} / 2 \\
& +(8 \sin \pi / 4)(\cos \pi / 4) \delta^{3} / 6+\ldots \\
& \text { higher order terms } \ldots \\
\sin \pi / 4=\cos \pi / 4 & =.707
\end{aligned}
$$

therefore,

$$
\cos ^{2}(\pi / 4+\delta)=1 / 2-\delta+0 \cdot \delta^{2}+0.67 \delta^{3}+\ldots
$$

Thus, the leading term in the correction to the linear approximation for $\cos ^{2} \theta_{1}-\cos ^{2} \theta_{2} \cong \theta_{2}-\theta_{1}=\delta_{2}-\delta_{1}$ is cubic in the angular difference $\left(\theta_{2}-\theta_{1}\right)$ expressed in radians.

For the maximum angular excursion reported in this investigation (.148 radians), the correction provided by the cubic term is only $1.5 \%$. Thus, in the worst case, the departure from linearity of $\left(\cos ^{2} \theta_{2}-\cos ^{2} \theta_{1}\right)$ is small and well within the limits of acceptable error rates.

2. We were initially concerned that the reexposure of the achromatic checks during the adaptation phase of Experiment 1 may have detracted from our effects (see Skowbo, 1974, and Jones \& Holding, 1975). The lack of differences in absolute magnitude of color aftereffects across Experiments 1, 2, and 3 indicates that this was not a problem. We might also point out here that the preadaptation matches for Experiments 1 and 2 were quite similar. The mean difference in angular deviation was $.66 \mathrm{deg}$ of rotation.

(Received for publication December 4, 1979; revision accepted July 15,1980 .) 